\title{
Parameterized Design and Analysis of Mechanical Properties of Three Hybrid Ellipsoidal Paraboloid Reticulated Shells
}

\author{
Xiaoyang Lu ${ }^{1, a}$, Xiaoxiao Wang ${ }^{1, b}$,Yaru Wang ${ }^{2, b}$,Hao Zhang ${ }^{2, b}$,Silu Xie ${ }^{2, b}$
}

${ }^{1}$ Research Institute of Engineering Mechanics, Shandong Jianzhu University, Jinan 250101, China

${ }^{2}$ Civil Engineering School, Shandong Jianzhu University, Jinan 250101,China

aluxy5504@163.com,1095313519@qq.com

Keywords:hybrid elliptical paraboloid latticed shell ; parametric design ; force performance comparison analysis

Abstract. Taking Changzhou Stadium Kiewitt-Lamella ellipsoid paraboloids as an example, three kinds of parametric design macros for hybrid ellipsoids paraboloid shells are developed using APDL parametric design language; The performance comparison of the three types of reticulated shells was performed using ANSYS software. The results show that: under the same working conditions, the structural form and force performance of the Kiewitt-Lamella ellipsoidal parabolic reticulated shell is the most reasonable, and it can be preferred in practical engineering; The three hybrid ellipsoid paraboloid cages have a better ratio of $1 / 4$ to $1 / 3$ than the $f / S$ ratio, which provides a good reference for the optimization of shape and engineering design of the reticulated shells.

\section{Introduction}

The hybrid ellipsoid parabolic reticulated shell improved the disadvantages of stress concentration near the apex of the single-type reticulated shell and the large number of connecting rods, so that the distribution of the mesh was uniform and the mechanical performance of the structure was improved [1]. The upper part of the ellipsoid parabolic roof of the Changzhou Stadium uses the K8 type, and the lower part uses the joint type hybridization [2]. In this paper, three kinds of ellipsoid paraboloid shells parametric design macro programs are developed by using APDL parametric design language, and ANSYS software is used to analyze its mechanical properties,some engineering conclusions are obtained.

\section{Geometric description of structure}

The five kinds of ellipsoid parabolic reticulated shells involved in this article are Rib-ring type, Schwedler, Kiewitt, Lamella and three-way lattice. To achieve structural hybridization, we need to satisfy: two structures On hybrid rings, the number of nodes in the two structures must be the same; The overall performance of hybridized shell structures should be optimized. Based on this, three kinds of hybrid ellipsoid paraboloid shells are established with the Kiewitt as the top structure: Kiewitt-Ribbed (KIE-RIB) and Kiewitt-Schwedler( KIE-SCH) and Kiewitt-Lamella(KIE-LAM).

There are seven main geometric parameters of hybrid ellipsoidal Paraboloid Latticed Shell, including long axis $S_{1}$, short axis $S_{2}$, vector height $f$, ratio coefficient $t$, circumferential symmetrical area number $K n$, total ring number of radial node $N x$ and the number of radial node rings of the superstructure $N s$ ( Fig 1). The ratio coefficient $t=N x-i /(N x-0.5)(i=1,2, \ldots N x)$ [3], according to the ratio coefficient $t$, the rise $f$ is divided and the projection coordinates $\mathrm{z}$ of each ciecle (or ring) on the vector height $f$ is obtained.

\section{Kiewitt-Lamella ellipsoid paraboloid latticed shell parametric design}

Reference spherical reticulated shell,Circular horizontal projection hybrid saddle shaped reticulated shelland rotating paraboloid reticulated shell parameterized design idea [4,5],parametric design macroprogram of kiewitt-lamella ellipsoidal paraboloid shell is developped. 
Given the long axis $S_{1}$, the short axis $S_{2}$ and the sagittal $f$ in the rectangular coordinate system, the elliptic parabolic equation is given:

$$
z=-4 f\left(\frac{x^{2}}{S_{1}^{2}}+\frac{y^{2}}{S_{2}^{2}}\right)+f
$$
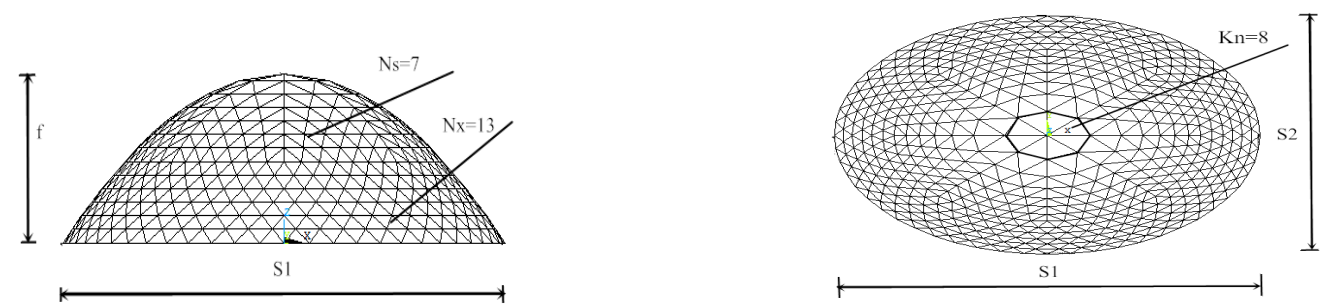

Fig.1 Kiewitt-Lamel hybrid ellipsoidal Paraboloid Latticed Shell macrogeometric parameters

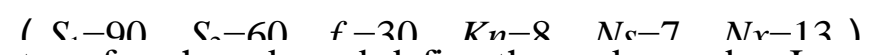

Calculate the coordinates of each node and define the node number:In spherical coordinates, first from the upper structure to discuss. The vertex is node 1 , coordinates $(0,0, f)$, according to the ratio coefficient $t$, The radial curve on the ellipsoidal paraboloid is divided so as to obtain the coordinates of each point of $\mathrm{X}, \mathrm{Y}, \mathrm{Z}$ the coordinate number for each node.

Rod connection:First, The upper structure of Kiewitt rod connection, when $i=1,2 \ldots N s, j=1$, $2 \ldots K n-1$,connecting $1+K n \times(i-1) \times i / 2+j$ and $1+K n \times(i-1) \times i / 2+j+1$ generates Circular pole of general symmetry zone,connecting $1+K n \times(i-1) \times i / 2+1$ and $1+K n \times(i-1) \times i / 2+K n \times i$ generates last symmetric region ring.connecting $1+K n \times(j-1) \times j / 2+1$ and $1+K n \times(j+1) \times j / 2+(k-1) \times(j+1)+i$ generates radial circumferential rod in the final symmetric region.connecting $1+K n \times(j-1) \times j / 2+(k-1) \times(j+1)+i$ and $1+K n \times(j+1) \times j / 2+(k-1) \times(j+1)+i$ generates unsymmetrical radial bar.Starting from the $N s+1$ lap, the connection of the rods is changed to Lamella.Using the same method and loop command,Set up the connection of the substructure rod.The last transition zone (between the $N s$ circle and the $N s+1$ circle) is connected by a bar, node $1+K n \times(N s-1) \times N s / 2+j$ and node $1+K n \times(N s-1) \times N s / 2+K n \times N s+j$ connection, connected by node $1+K n \times(N s-1) \times N s / 2+j$ and $1+K n \times(N s-1) \times N s / 2+K n \times N s+j+1$ Generic symmetry zone member connection is generated, $1+K n \times(N s-1) \times N s / 2+K n \times N s$ and $1+K n \times(N s-3) \times N s / 2$ generate the last symmetry zone The rods are connected, the last $1+K n \times(N s-1) \times N s / 2+K n \times N s$ and $1+K n \times(N s-1) \times N s / 2+K n \times N s+1$ connections. That is, Kiewitt-Lamella hybrid ellipsoid paraboloid shell is formed.

\section{Selection of structural materials and model load}

The Q235 hot rolled seamless bars are used in the structure. The density is $7850 \mathrm{~kg} / \mathrm{m}^{3}$. The modulus of elasticity is $2.06 \times 10^{5} \mathrm{MPa}$.The Poisson ratio is 0.3 . The element of beam4 in the ansys is used in the modeling [6].The rigid joints are ideal.The hinged supports are used in the structure which has 1/400 [7]with span of structural allowable displacement and $215 \mathrm{MPa}$ of allowable stress. The constitutive relation of structural material is an ideal elastic-plastic material without consideration of material reinforcement. The hinges are applied to the outer ring node of the reticulated shell (only limit the three directional lines of $\mathrm{x}, \mathrm{y}$ and $\mathrm{z}$, without restricting rotation), structural self weight (including rod and node) is Considered, The outer load of the roof is $2.35 \mathrm{KN} / \mathrm{m}^{2}$ [8].Bar section is $\Phi 114 \times 4.0$.

\section{The Influence of the Number of $N_{S}$ of Radial Nodes in Superstructure on the Static Performance of Reticulated Shell}

Select $S_{1}=80 \mathrm{~m}, S_{2}=50 \mathrm{~m}, f=30 \mathrm{~m}, f / S=3 / 8, K n=8, N x=14, N s=4 \sim 10$ to analyze the static performance of three ellipsoid paraboloid shells The maximum displacement value and the minimum stress value corresponding to the reticulated shells varied with $N s$, as shown in Table 1 and Table 2. The maximum deflection of the structure is $S_{2} / 400=0.125 \mathrm{~m}$ and the allowable stress is $215 \mathrm{MPa}$. 
From Tables 1 and 2, we know: (1) The maximum displacement of the three type reticulated shell first decreases and then increases with increasing $N_{S}$. In addition to the trend of KIE-RIB, the most unfavorable stress decreases with the increase of $N_{S}$; the larger the number of radial turns in the form of rib rings and joints, the overall stiffness of the structure is weaker, and thus fully reflected from the side The knight pattern is more rigid than the other three forms.

(2) When $N_{S}=5 \sim 10$, KIE-LAM is the best performance and the three kinds of hybrid elliptic parabolic reticulated shell is suitable for $N_{S}=7 \sim 9$.

Table 1 The maximum displacement corresponding to different values of the number of radial turns $N_{S}$ in the upper structure $(\mathrm{cm})$

\begin{tabular}{cccccccc}
\hline$N_{\mathrm{S}}$ & 4 & 5 & 6 & 7 & 8 & 9 & 10 \\
\hline KIE-RIB & 14.81 & 9.13 & 5.89 & 3.88 & 2.69 & 2.10 & 1.84 \\
KIE-SCH & 2.48 & 2.09 & 1.82 & 1.64 & 1.62 & 1.73 & 1.80 \\
KIE-LAM & 3.85 & 2.54 & 1.99 & 1.75 & 1.65 & 1.63 & 1.66 \\
\hline
\end{tabular}

Table 2 Maximum stress corresponding to different values of the number of radial turns $N_{S}$ in the upper structure $(\mathrm{MPa})$

\begin{tabular}{cccccccc}
\hline$N_{S}$ & 4 & 5 & 6 & 7 & 8 & 9 & 10 \\
\hline KIE-RIB & - & -213 & -167 & -135 & -120 & -108 & -98.7 \\
KIE-SCH & -211 & -174 & -149 & -131 & -117 & -108 & -101 \\
KIE-LAM & -197 & -144 & -112 & -90.2 & -74.9 & -65.3 & -66.3 \\
\hline
\end{tabular}

Note: - Indicates that the value exceeds the allowable value, the same as below.

\section{Influence of the Number of $K n$ in the Area of the Loop on the Static Performance of the Reticulated Shell}

$S_{1}=80 \mathrm{~m}, S_{2}=50 \mathrm{~m}, f=30 \mathrm{~m}, f / S=3 / 8, N x=14, K n=6 \sim 18$ (step size is 2 ), $N s=9$ ellipsoid paraboloid shell is obtained. The maximum displacement value and the minimum stress value corresponding to $\mathrm{Kn}$ change, reticulated shell, are shown in Table 3 and Table 4 . The maximum deflection of the structure is $S_{2} / 400=0.125 \mathrm{~m}$ and the allowable stress is $215 \mathrm{MPa}$.

Table 3 Maximum displacement corresponding to different values of the number Kn of the circumferential region $(\mathrm{cm})$

\begin{tabular}{cccccccc}
\hline$K n$ & 6 & 8 & 10 & 12 & 14 & 16 & 18 \\
\hline KIE-RIB & 2.21 & 2.10 & 1.77 & 1.68 & 1.50 & 1.46 & 1.40 \\
KIE-SCH & 1.80 & 1.73 & 1.61 & 1.60 & 1.51 & 1.52 & 1.47 \\
KIE-LAM & 1.63 & 0.01 .6 & 1.49 & 1.52 & 1.40 & 1.42 & 1.38 \\
\hline
\end{tabular}

Table 4 Maximum stress corresponding to different values of the number Kn of the circumferential zone number(MPa)

\begin{tabular}{cccccccc}
\hline$K n$ & 6 & 8 & 10 & 12 & 14 & 16 & 18 \\
\hline KIE-RIB & -137 & -108 & -89.1 & -76.4 & -68.1 & -60.9 & -60.2 \\
KIE-SCH & -134 & -108 & -89.4 & -77.5 & -67.6 & -61.8 & -61.5 \\
VI__Ad & -92.4 & -65.3 & -55.5 & -56.7 & -59.4 & -62.1 & -63.8 \\
\hline
\end{tabular}

From Tables 3 and 4, we know that with the increase of $K n$, in addition to KIE-LAM, the most unfavorable stress and maximum displacement show a decreasing law of uniform change. When $K n=6$ is increased to 18 , the most unfavorable displacement is reduced in millimeters, and $K n$ is more sensitive to the most adverse stress effect of the three kinds of reticulated shell. $K n$ is recommended to take 6 14. 


\section{The influence of vector height $\boldsymbol{f}$ on the static performance of reticulated shell}

Select $S_{1}=80 \mathrm{~m}, S_{2}=50 \mathrm{~m}, f=12 \sim 40$ (step size is 4 ), $N x=14, K n=8, N s=9$ ellipsoid paraboloid reticulated shell for static performance analysis and obtain the change with $f$ The maximum and minimum stress values corresponding to the reticulated shells are shown in Tables 5 and 6 . The maximum deflection of the structure is $S_{2} / 400=0.125 \mathrm{~m}$ and the allowable stress is $215 \mathrm{MPa}$.

From Table 5, 6:With the increase of $f$, the maximum displacement and the most unfavorable stress first decreased and then increased. The maximum displacement and the most unfavorable stress of KIE-RIB at $f=20$ reached the minimum value. When $f=20$ increased to $f=28$, the maximum displacement of KIE-LAM increased by $6.08 \%$, the most unfavorable The stress is reduced by $10.79 \%$, and $f$ is more sensitive to the most unfavorable stress. It is recommended that the three kinds of reticulated shells have a $f / S$ ratio of $1 / 4$ to $1 / 3$.

Table 5 Maximum displacement corresponding to different values of vector height $f(\mathrm{~cm})$

\begin{tabular}{cccccccc}
\hline$f$ & 12 & 16 & 20 & 24 & 28 & 32 & 40 \\
\hline KIE-RIB & 2.97 & 1.88 & 1.40 & 1.57 & 1.91 & 2.32 & 3.37 \\
KIE-SCH & 2.41 & 1.77 & 1.54 & 1.59 & 1.68 & 1.79 & 2.14 \\
KIE-LAM & 2.42 & 1.55 & 1.48 & 1.49 & 1.57 & 1.71 & 2.06 \\
\hline
\end{tabular}

Table 6 Maximum stress corresponding to different values of vector height $f(\mathrm{MPa})$

\begin{tabular}{cccccccc}
\hline$f$ & 12 & 16 & 20 & 24 & 28 & 32 & 40 \\
\hline KIE-RIB & -129 & -105 & -99.8 & -102 & -106 & -111 & -122 \\
KIE-SCH & -132 & -117 & -111 & -108 & -107 & -108 & -117 \\
KIE-LAM & -111 & -87.4 & -73.2 & -66.2 & -65.3 & -65.8 & -69.4 \\
\hline
\end{tabular}

\section{Conclusions}

In this paper, a parametric design macro program of three hybrid ellipsoid paraboloid shell is developed. The ANSYS software is used to analyze the parameters of vector heights, circumferential area scores, number of radial node turns.Performance analysis shows that:

According to the actual engineering design, the relevant geometric parameters need to be input. The parametric design macro program of the hybrid ellipsoid paraboloid shell can be used to form the required calculation and analysis model; this macro program is easy to use and is the optimal design for this kind of lattice shell structure, Mechanical properties analysis and building selection provide convenience.

In the three types of structures, under the same conditions, the structure and force performance of the Kiewitt-Lamella reticulated shell are the most reasonable and can be preferred when designing the actual engineering.

\section{Acknowledgements}

This work was financially supported by projects of Shandong postgraduate innovation (SDYY08038) and postgraduate high quality courses of Shandong Jianzhu University (YZKC201605).

\section{References}

[1] Xiaoyang Lu, Xiaowei Zhao, Shiying Chen.Discrete Variable Lattice Structure Optimization Design. Beijing: China Architecture \& Building Press, 2013.

[2] Bin Luo, Zhengxing Guo, Yuan Feng.Study on the stability of chord arch dome of elliptical paraboloid in Changzhou Stadium. Journal of Building Structures, 2009, 30(6): 148-154.

[3] Jing Xin , Xiaoyang Lu.Parametric Design of Single-layer Ellipsoidal Reticulated Shell. Low Temperature Architecture Technology, 2014, 36(12):53-55.

[4] Ya Su,Shao bo Lu,Xiaoyang Lu.Parametric modeling and shape optimization of hybrid saddle reticulated shell.Journal of Shandong Jianzhu University,2016,01:38-46. 
[5] Yaruo Chen,Shaobo Lu,Xiaoyang Lu.The fusion of traditional architectural style and modern lattice shell structure.Shandong Architecture University Journal, 2014.29(1):35.

[6] Xinmin Wang.Numerical Analysis of ANSYS Engineering Structure. China Communications Press, 2007.

[7] GB 50009-2012 Load code for the design of building structures.Beijing:China Architecture\&Building Press,2012.

[8] JGJ7-2010 Spatial Grid Structure Specification. Beijing: China Building Industry Press, 2010. 